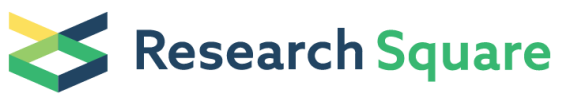 \\ Preprints are preliminary reports that have not undergone peer review. \\ They should not be considered conclusive, used to inform clinical practice, \\ or referenced by the media as validated information.
}

\section{Postoperative Concurrent Chemoradiotherapy Plus Apatinib for First-line Treatment of Patients with Malignant Glioma: From One Single Research Institute}

\author{
Zhichao FU ( $\sim$ fauster1112@126.com ) \\ The 900th hospital of the Joint Logistics Team https://orcid.org/0000-0003-0341-106X \\ Xiaoyan Li \\ Fuzong Clinical Medical College of Fujian Medical University \\ Wenmin YING \\ The 900th Hospital of the Joint Logistics Team \\ Ivjuan CAI \\ The 900th Hospital of the Joint Logistics Team \\ Guo LI \\ The 900th Hospital of the Joint Logistics Team \\ Huachun LUO \\ The 900th Hospital of the Joint Logistics Team \\ Xinpeng WANG \\ Fuzong Clinical Medical College of Fujian Medical University \\ Mengjing WU \\ Fuzong Clinical Medical College of Fujian Medical University \\ Jing FENG \\ The 900th Hospital of the Joint Logistics Team
}

\section{Research Article}

Keywords: antiangiogenic agents, apatinib, malignant glioma

Posted Date: April 19th, 2021

DOI: https://doi.org/10.21203/rs.3.rs-404177/v1

License: (c) (1) This work is licensed under a Creative Commons Attribution 4.0 International License. Read Full License 


\section{Abstract}

Objective: To explore the effectiveness and safety of apatinib in patients with malignant glioma.

Methods: This is a retrospective case-control study in a single center. Patients with new postoperative pathological diagnosis of malignant glioma (WHO 『 ه) were selected. Enrolled patients received concurrent chemoradiotherapy (60Gy/30f/6w;

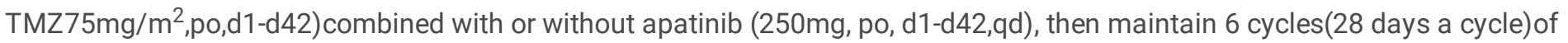
TMZ chemotherapy $\left(200 \mathrm{mg} / \mathrm{m}^{2}, \mathrm{~d} 1-\mathrm{d} 5\right)$. The primary endpoints were progression free survival (PFS) and the grade of peritumoral brain edema (PTBE) evaluated by edema index(EI). The secondary endpoint was overall survival(OS). Hazard ratios of PFS and OS were compared between trials in a Cox proportional hazards model.

Results: 48 patients (24 in apatinib group and 24 in control group) were enrolled in this study. The results elucidated that the mPFS of the apatinib group was longer than control group, but the difference was not statistically significant( 9.63 vs. 7.33 months; $P=0.073)$.As for mOS, the results of two groups were almost similar.(15.47 vs. 14.70 months, $P=0.612)$. Cox multivariate regression model revealed that Apatinib was not a prognostic factor for PFS and OS ( $>0.05)$. Multivariate analysis showed that tumor grade was an important risk factor for PFS and OS.The grade of PTBE was improved in 15 of 23 patients (65.2\%) in apatinib group versus 6 of $24(25 \%)$ in control group. There was no grade 3 or 4 adverse events and serious adverse events.

Conclusion: Apatinib group can improve mPFS by 2.3 months in patients with malignant glioma,but there was no statistical significance $(\mathrm{P}>0.05)$. The results also indicated that apatinib conferred a significant beneficial effect on PTBE improvement. All occurred adverse reactions were moderate and controllable.

\section{Background}

Glioma is currently the tumor with the highest incidence rate of the central nervous system. Glioblastoma is the most malignant and invasive among gliomas, accounting for about $48.3 \%$ of primary malignant brain tumors.And the 5 -year survival rate is only $6.8 \%$. Although surgery, radiotherapy, chemotherapy, and targeted therapies are currently feasible, the median overall survival (OS) time of glioblastoma is only about 14.4 months.

In 2005, the European Cancer Research and Treatment Collaboration Group and the National Cancer Institute of Canada (EORTC / $\mathrm{NCIC}$ ) reported a phase III clinical study,which found that in newly diagnosed GBM patients,the median progression-free survival (PFS) and median overall survival of patients who were treated with concurrent chemoradiotherapy with TMZ plus 6 cycles of TMZ adjuvant therapy were significantly prolonged than that of patients underwent radiotherapy alone.The 5-year survival rates were $1.9 \%$ and $9.8 \%$, respectively, and the hazard ratio was $0.63(95 \% \mathrm{The} \mathrm{Cl}$ is $0.5-0.7, \mathrm{P}<0.0001)$. Accordingly, concurrent chemoradiotherapy with TMZ and subsequent adjuvant therapy have become the standard treatment for newly diagnosed GBM.

As we know,tumor angiogenesis plays a crucia and complex role in the pathogenesis of tumorigenesis, progression,recurrence and metastasis.Studies have demonstrated that many pathways are engaged in the formation of tumor angiogenesis. Therefore, in the treatment of advanced tumors, anti-angiogenic factor therapy has been proposed at home and abroad.Malignant glioma is one of the tumors with high degree of vascularization in human body. The growth, invasion and malignant progression of tumors correlate fairly with angiogenesis. Among them, VEGF is not only the main regulator of tumor angiogenesis, but also the strongest angiogenesis factor.

Apatinib mesylate tablets is a small molecule tyrosine kinase inhibitor,targeting vascular endothelial growth factor receptor 2 (VEGFR-2), which can block the binding of VEGF to its receptor, inhibit the generation of new blood vessels in tumor tissues and achieve the goal of suppressing tumor growth.And it is clinically proven to tolerability, safety and effectiveness.

Therefore, based on the limited data at present,we designed such a research scheme which combines anti-angiogenic drugs with concurrent chemoradiotherapy, aiming to explore a new treatment for postoperative patients with malignant glioma. Through this study,we will further confirm the effectiveness and safety of anti-angiogenic drugs for malignant gliomas, and provide guidance for future clinical medication. 


\section{Materials And Methods \\ 2.1 study design}

This single-center, retrospective, case-control study explored the efficacy and safety of apatinib plus chemoradiotherapy with temozolomide for postoperative patients with malignant glioma.

The primary endpoints were investigator-assessed PFS and the improvement of PTBE, and the secondary endpoints were OS and safety. In this study,investigator referred to PFS as the period from the first day of radiotherapy to the occurrence of disease progression or death because of any cause, whichever occurred first. OS was defined as the period from the first day of radiotherapy to death due to any cause.

The evaluation of PTBE can be divided into the following categories: No edema: no obvious edema area can be observed by imaging examination methods such as MRl;Mild edema: the width of edema area $\leq 2 \mathrm{~cm}$; Moderate edema: the width of edema area $<3 \mathrm{~cm}$; Severe edema: the width of edema area $>3 \mathrm{~cm}$.No edema, mild edema, moderate edema and severe edema were respectively recorded as 0, 1, 2 and 3 points,namely edema index. And the average edema index was calculated before and after treatment, respectively.The major safety indexs were vital signs, laboratory indicators, adverse events (AE), severe adverse events (SAE), and specific adverse events (e.g., hypertension, proteinuria, and hand-foot syndrome).

The trial was reviewed by our center's ethics committee and registered on Clinical Trials.gov with number NCTxxxxxxxx.

\subsection{Eligible criteria}

2.2.1 Eligible patients were $\geq 18$ years old and $\leq 70$ years old with histopathologically confirmed malignant glioma (World Health Organization [WHO] Grade III or IV).All had received no more than one surgery and not received radiotherapy within 3 months before admission. The estimated survival period had to be more than 3 months.Additional inclusion criteria were Eastern Cooperative Oncology Group (ECOG) physical status score of $0-1$,adequate hematologic, renal and hepatic function, that is, the following requirements were met in the week before enrollment:Hemoglobin $>100 \mathrm{~g} / \mathrm{L}$ (no transfusion within 14 days), neutrophil count $>1.5 \times 109 / \mathrm{L}$, platelet count $>75 \times 109 / \mathrm{L}$, total bilirubin $\leq 1.5 \times \mathrm{ULN}$ (upper limit of normal value), alanine aminotransferase (ALT) and aspartate aminotransferase(AST) $\leq 3.0 \times U L N$, and endogenous creatinine clearance $\geq 60 \mathrm{ml} / \mathrm{min}$ (Cockcroft-Gault formula).

2.2.2 Patients with one of the following conditions were excluded:those with multiple factors that affect oral medication (such as dysphagia, chronic diarrhea and intestinal obstruction, etc.); those with 2 grand mal epilepsy in the previous week or $\geqq 3$ times in the previous month,those with a history of haemorrhagic disease, and any bleeding event with severe grade 3 or above in CTCAE4.0 occurred within 6 months before screening;those with a clear tendency of gastrointestinal bleeding, such as severe varicosis or coagulation dysfunction (INR > 1.5, APTT > 1.5 ULN); patients with hypertension who cannot be well controlled by single anti-hypertensive drug (systolic pressure $>140 \mathrm{mmHg}$, diastolic pressure $>90 \mathrm{mmHg}$ ), those newly diagnosed as angina pectoris within 3 months before screening or those with myocardial infarction within 6 months before screening,those with arrhythmia (including QTc: male $\geq 450 \mathrm{~ms}$, female $\geq 470 \mathrm{MS}$ ) required long-term use of anti-arrhythmic drugs and New York Heart Association grade $\geq \mathrm{II}$ cardiac insufficiency;those with positive urine protein (urine protein test $2+$ or more, or 24-hour urine protein quantity $>1.0 \mathrm{~g}$ ). In addition,for female subjects, they should be sterilized by operation,or postmenopausal or they agreed to use a medically recognized contraceptive measure during the study treatment period and within the first 6 months after the end of the study treatment period,the serum or urine pregnancy test must be negative for 7 days prior to study enrollment and must be nonlactating.For male subjects, they should be sterilized by operation,or they agreed to use a medically recognized contraceptive measure during the study treatment period and within the first 6 months after the end of the study treatment period.According to the judgment of the investigator, patients had concomitant diseases that seriously endanger the safety or affect the completion of the study.

\subsection{Treatment}

A total of 47 patients were screened in this study from October 2017 to March 2019, including 23 patients in the apatinib group and 24 patients in the control group.The median age was 47 years (range 19-73 years) versus 48 years (range 24-68 years).

Page 3/14 


\subsubsection{Concurrent radiochemotherapy}

Concurrent radiotherapy: GTV was defined as the tumor visible on enhanced MR or other imaging methods; GTVtb was defined as the operative cavity judged by preoperative and postoperative enhanced MR image; CTV1 was GTV or GTVtb + 1.5-2cm; CTV2 was GTV or GTVtb + 2-2.5cm; PTV1 and PTV2 were expanded $0.3 \mathrm{~cm}$ on the basis of CTV1 and CTV2; the irradiation dose of PTV1 and PTV2 were 60Gy and 54Gy respectively (5 times per week, a total of 30 times).

Concurrent chemotherapy: the control group regimen: low-dose temozolomide at 75mg/m2, d1-d42,the experimental regimen: lowdose temozolomide at $75 \mathrm{mg} / \mathrm{m} 2$, d1-d42,combined with apatinib mesylate, $250 \mathrm{mg}$ qd,from the first day of radiotherapy to the end of, about 6 weeks.

\subsubsection{Maintenance treatment}

Adjuvant therapy began 1 month after the end of concurrent radiochemotherapy. The control group: TMZ: 200mg/m2.d1-5, every 28 days as a treatment cycle, a total of 6 cycles. The experimental group: apatinib mesylate,250mg,d1-28; TMZ: 200mg/m2.d1-5, every 28 days as a treatment cycle, a total of 6 cycles.

\subsection{Statistical analysis}

SPSS version 16.0 was used for statistical analysis. $P<0.05$ indicates that the difference is statistically significant. Regarding whether or not to use corticosteroids, we utilized the chi-square test to perform an analysis. In addition, wilcoxon test was used to compare the changes of peritumoral edema before and after treatment between the two groups. Survival was estimated using the Kaplan-Meier method. We used the log-rank test to analyze the factors of survival time for any significant differences. Predictors of clinical radioresistance were identified by logistic regression analysis. Cox's regression analysis was used to calculate the prognostic significance of individual parameters.

\section{Results}

\subsection{Patients}

From October 2017 through May 2020, a total 48 patients were enrolled at a single clinical center, 24 patients in the apatinib group and 24 patients in the control group (Fig. 1). The baseline characteristics of the patients were well balanced between the two groups (Table 1).

Table.1 Baseline demographic and clinical characteristics 


\begin{tabular}{|c|c|c|c|}
\hline Characteristics & Apatinib group & Control group & $\mathrm{P}$ Value \\
\hline Median age,yrs(IQR) & $48(42-53)$ & $49(44-54)$ & 0.62 \\
\hline Male/female,n(\%) & 16(66.67)/8(33.33) & $15(62.50) / 9(37.50)$ & 0.76 \\
\hline ECOG PS $0 / \geq 1, n(\%)$ & $15(62.50) / 9(32.50)$ & $11(45.83) / 13(54.17)$ & 0.25 \\
\hline Median time since surgery,d(IQR) & $33(26-38)$ & $26(20-32)$ & 0.78 \\
\hline \multicolumn{4}{|l|}{ Surgical status } \\
\hline Total resection,n(\%) & $9(37.50)$ & $10(41.67)$ & \multirow[t]{3}{*}{0.77} \\
\hline Subtotal resection,n(\%) & $11(45.83)$ & $9(37.50)$ & \\
\hline Partial resection, $\mathrm{n}(\%)$ & $4(16.67)$ & $5(20.83)$ & \\
\hline \multicolumn{4}{|l|}{ Pathological grading(WHO) } \\
\hline 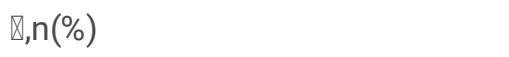 & $6(25.00)$ & $7(29.17)$ & \multirow[t]{2}{*}{0.75} \\
\hline$\bigotimes, \mathrm{n}(\%)$ & 18(75.00) & 17(70.83) & \\
\hline \multicolumn{4}{|l|}{ IDH status } \\
\hline Unmutated,n(\%) & $5(20.83)$ & $11(66.67)$ & \multirow[t]{3}{*}{0.16} \\
\hline Mutated,n(\%) & 18(75.00) & $8(25.00)$ & \\
\hline Unknown,n(\%) & $1(4.17)$ & $5(8.33)$ & \\
\hline \multicolumn{4}{|l|}{ MGMT status } \\
\hline Unmutated,n(\%) & $7(29.17)$ & $2(8.33)$ & \multirow[t]{3}{*}{0.10} \\
\hline Mutated,n(\%) & $16(66.66)$ & $18(75.00)$ & \\
\hline Unknown,n(\%) & $1(4.17)$ & $4(16.67)$ & \\
\hline
\end{tabular}

\subsection{Efficacy Outcomes}

The median PFS was 9.63 months in the apatinib group as compared with 7.33 months in the control group, but the difference was not statistically significant.(hazard ratio, $0.55 ; 95 \%$ confidence interval [Cl], 0.29 to $1.07 ; P=0.073$ )(Fig. 1).Survival analysis using Cox multivariate regression model revealed that there were no independent risk factors for PFS $(P>0.05)$ although PFS seemed to be prolonged in the apatinib group.In multivariate analysis, tumor grade was an important risk factor for PFS after adjustment for baseline stratification (hazard ratio, 4.013;95\% $\mathrm{Cl}, 1.067$ to $15.093 ; \mathrm{P}=0.040$ ) (Table 3 ).

For median overall survival, the results of two groups were almost similar. The median OS was 15.47 months in the apatinib group versus 14.70 months in the control group(15.47 months vs. 14.70 months; hazard ratio, $0.84 ; 95 \% \mathrm{Cl}, 0.43$ to $1.66 ; \mathrm{P}=0.612$ ) (Fig. 2). Survival analysis using Cox multivariate regression model revealed that age and tumor grade was an independent prognostic factor for overall survival (P区0.05) .In multivariate analysis, tumor grade was an important risk factor for PFS after adjustment for baseline stratification (hazard ratio, $5.211 ; 95 \% \mathrm{Cl}, 1.216$ to $22.416 ; \mathrm{P}=0.026$ )(Table 3 ).

In control group,the median PFS was 7.33 months, and the 1-year PFS was $29.2 \%$. The median OS was 14.7 months, and the 12 month and 24-month OS rates were 75\% and 13.6\%.Among apatinib group,the median PFS was 9.63 months and the 1-year PFS was $42.6 \%$. The median OS was 15.47 months, and the 12 -months and 24 -months OS rate were $79.9 \%$ and $22.6 \%$. The values of PFS and OS are shown in Table 2.

As of the statistical date, there were 7 patients with stable disease (SD), 16 patients with progressive disease (PD) and 13 patients with death in the apatinib group; whereas in the control group, the condition of 3 patients remained stable, the other 21 patients had progressed, with 19 unfortunate deaths. Pseudo progression was observed in 1 patient in the Apatinib group and not in the control group. The cause of death in all patients was due to disease progression and no other causes of death were observed. 
Table 2

The PFS and OS rate of two groups

\begin{tabular}{|llll|}
\hline & Apatinib group & Control group & P Value \\
\hline PFS rate(\%) & & & \\
\hline Median & 9.63 & 7.33 & \\
\hline 3-month(\%) & 100 & 91.7 & NE \\
\hline 6-month(\%) & 91.7 & 58.3 & 0.515 \\
\hline 12-month(\%) & 42.6 & 29.2 & 0.057 \\
\hline 18-month(\%) & 28.4 & 4.2 & 0.502 \\
\hline 24-month(\%) & 28.4 & 4.2 & 0.171 \\
\hline OS rate(\%) & & & \\
\hline Median & 15.47 & 14.70 & \\
\hline 6-month(\%) & 100 & 100 & NE \\
\hline 12-month(\%) & 79.9 & 75 & 0.022 \\
\hline 18-month(\%) & 37.7 & 31.8 & 0.633 \\
\hline 24-month(\%) & 22.6 & 13.6 & 0.933 \\
\hline
\end{tabular}


Table 3

Cox proportional hazards model of factors to predict PFS and OS

\begin{tabular}{|c|c|c|c|c|c|c|c|c|}
\hline & \multicolumn{4}{|l|}{ PFS } & \multicolumn{4}{|l|}{ os } \\
\hline & \multicolumn{2}{|c|}{ Univariate Analysis } & \multicolumn{2}{|c|}{ Multivariate analysis } & \multicolumn{2}{|c|}{ Univariate Analysis } & \multicolumn{2}{|c|}{ Multivariate analysis } \\
\hline & $\mathrm{HR}(95 \% \mathrm{Cl})$ & $\begin{array}{l}\mathrm{P} \\
\text { Value }\end{array}$ & $\mathrm{HR}(95 \% \mathrm{Cl})$ & $\begin{array}{l}\mathrm{P} \\
\text { Value }\end{array}$ & $\begin{array}{l}\mathrm{HR}(95 \% \\
\mathrm{Cl})\end{array}$ & $\begin{array}{l}\mathrm{P} \\
\text { Value }\end{array}$ & $\mathrm{HR}(95 \% \mathrm{Cl})$ & $\begin{array}{l}\mathrm{P} \\
\text { Value }\end{array}$ \\
\hline \multicolumn{9}{|l|}{ Group } \\
\hline Control & 1 & & - & - & 1 & & - & - \\
\hline \multirow[t]{2}{*}{ apatinib } & 0.053 & 0.073 & - & - & 0.839 & 0.612 & - & - \\
\hline & $\begin{array}{l}(0.287- \\
1.066)\end{array}$ & & & & $\begin{array}{l}(0.426- \\
1.655)\end{array}$ & & & \\
\hline \multicolumn{9}{|l|}{ Age } \\
\hline$<50$ & 1 & & - & - & 1 & & - & - \\
\hline \multirow[t]{2}{*}{$\geq 50$} & 1.730 & 0.094 & - & - & 2.525 & 0.011 & - & - \\
\hline & $\begin{array}{l}(0.911- \\
3.285)\end{array}$ & & & & $\begin{array}{l}(1.232- \\
5.174)\end{array}$ & & & \\
\hline \multicolumn{9}{|l|}{ Surgical status } \\
\hline Incomplete resection & 1 & & - & - & 1 & & - & - \\
\hline \multirow[t]{2}{*}{ Total resection } & 0.762 & 0.421 & - & - & 0.885 & 0.734 & - & - \\
\hline & $\begin{array}{l}(0.393- \\
1.479)\end{array}$ & & & & $\begin{array}{l}(0.437- \\
1.794)\end{array}$ & & & \\
\hline \multicolumn{9}{|l|}{$\begin{array}{l}\text { Pathological } \\
\text { grading(WHO) }\end{array}$} \\
\hline ૧ & 1 & & 1 & & 1 & & 1 & \\
\hline \multirow[t]{2}{*}{ ૫ } & 2.088 & 0.068 & $\begin{array}{l}4.013(1.067- \\
15.093)\end{array}$ & 0.040 & 2.864 & 0.021 & $\begin{array}{l}5.211(1.216- \\
22.416)\end{array}$ & 0.026 \\
\hline & $\begin{array}{l}(0.946- \\
4.610)\end{array}$ & & & & $\begin{array}{l}(1.171- \\
7.033)\end{array}$ & & & \\
\hline \multicolumn{9}{|l|}{ IDH status } \\
\hline Unmutated & 1 & & - & - & 1 & & - & - \\
\hline \multirow[t]{2}{*}{ Mutated } & 0.564 & 0.156 & - & - & 0.329 & 0.025 & - & - \\
\hline & $(0.244-1.300)$ & & & & $\begin{array}{l}(0.125- \\
0.870)\end{array}$ & & & \\
\hline \multicolumn{9}{|l|}{ MGMT status } \\
\hline Unmutated & 1 & & - & - & 1 & & - & - \\
\hline \multirow[t]{2}{*}{ Mutated } & 1.538 & 0.276 & - & - & 1.640 & 0.229 & - & - \\
\hline & $\begin{array}{l}(0.694- \\
3.409)\end{array}$ & & & & $\begin{array}{l}(0.713- \\
3.769)\end{array}$ & & & \\
\hline \multicolumn{9}{|l|}{ Gender } \\
\hline Male & & & - & - & 1 & & - & - \\
\hline
\end{tabular}




\begin{tabular}{|c|c|c|c|c|c|c|c|c|}
\hline & PFS & & & & OS & & & \\
\hline \multirow[t]{2}{*}{ Female } & 0.993 & 0.984 & - & - & 0.750 & 0.423 & - & - \\
\hline & $\begin{array}{l}(0.512- \\
1.928)\end{array}$ & & & & $\begin{array}{l}(0.375- \\
1.501)\end{array}$ & & & \\
\hline \multicolumn{9}{|c|}{ ECOG PS } \\
\hline 0 & & & - & - & 1 & & - & - \\
\hline \multirow[t]{2}{*}{$\geq 1$} & 1.657 & 0.121 & - & - & 1.152 & 0.68 & - & - \\
\hline & $\begin{array}{l}(0.875- \\
3.138)\end{array}$ & & & & $\begin{array}{l}(0.588- \\
2.256)\end{array}$ & & & \\
\hline
\end{tabular}

\subsection{Results of peritumoral brain edema (PTBE)}

The grade of PTBE was improved in 15(65.2\%) of 23 patients and stable in 8(34.8\%) patients in apatinib group, and corticosteroids were used in $5(21.7 \%)$ of 23 patients. In the control group, the grade of PTBE was improved in $6(25 \%)$ of 24 patients, $16(66.7 \%)$ was stable,2(8.3\%) was aggravated, and $14(58.3 \%)$ of 24 patients in this group were previously on corticosteroids. Regarding whether or not to use corticosteroids, we utilized the chi-square test to perform an analysis.P value was 0.02393 , which is considered to be statistically different.In addition, wilcoxon test was used to compare the changes of peritumoral edema before and after treatment between the two groups, in which the $P$ value was 0.0007593 , with a statistically significant difference.

The following is the data of a representative case.A 42-years-old male patient presented with the chief complaint of "headache for 2 years, aggravation and vomiting for 1 day". MRI examination showed a mass in the left frontotemporal lobe with obvious edema zone on September 26, 2017 (Fig. 3.). He received tumor resection On September 28, 2017, and the postoperative histopathology showed glioblastoma. MRI examination showed postoperative changes in the left frontotemporal lobe and PTBE in the range of > 3cm on September 30, 2017 (Fig. 3) .From October 19, 2017 to November 30, 2017, he was treated by combination of postoperative concurrent chemoradiotherapy with TMZ 120mg/d and anti-vascular therapy with apatinib 250mg/d,then 6 cycles of maintenance therapy with TMZ $350 \mathrm{mg} / \mathrm{d}$. MRI examination showed PTBE in the range of $<1 \mathrm{~cm}$, which was significantly smaller than before,on December 26, 2017( Fig. 3). The patient died on June 17, 2020 with a PFS of 13.3 months and an OS of 32.4 months.

\subsection{Toxicity and adverse events}

The adverse events(AEs) observed in the study were listed in Table 4.It was reported that $69.6 \%$ of patients in apatini group and $50.0 \%$ of patients in control group had AEs of any degree. The grade 1 and grade 2 adverse reactions were $68.8 \%$ and $31.2 \%$, respectively, in the Apatinib group. While the grade 1 and grade 2 adverse reactions accounted for $58.3 \%$ and $41.7 \%$ in the control group, respectively.Hypertension, hand-foot syndrome and proteinuria were the special adverse reactions in apatinib group.There was no grade 3 or 4 AEs and unanticipated AEs.After symptomatic treatment, these AEs were rapidly reduced and recovered without any reduction in drug dose or interruption. No serious adverse events occurred in the study and all adverse reactions were controllable. 
Table 4

Adverse events in two groups.

\begin{tabular}{|c|c|c|c|c|c|c|c|c|c|c|}
\hline \multirow{2}{*}{$\begin{array}{l}\text { Adverse } \\
\text { events }\end{array}$} & \multicolumn{2}{|c|}{ Grade $1(n, \%)$} & \multicolumn{2}{|c|}{ Grade 2(n,\%) } & \multicolumn{2}{|c|}{ Grade $3(n, \%)$} & \multicolumn{2}{|c|}{ Grade 4(n,\%) } & \multicolumn{2}{|c|}{ Total $(n, \%)$} \\
\hline & Apatinib & Control & Apatinib & Control & Apatinib & Control & Apatinib & Control & Apatinib & Control \\
\hline $\begin{array}{l}\text { Myelosuppre- } \\
\text { ssion }\end{array}$ & $1(4.3)$ & 0 & 0 & $1(4.3)$ & 0 & 0 & 0 & 0 & $1(4.3)$ & $1(4.3)$ \\
\hline $\begin{array}{l}\text { Gastrointesei- } \\
\text { nal reaction }\end{array}$ & $2(8.7)$ & $3(12.5)$ & $1(4.3)$ & 0 & 0 & 0 & 0 & 0 & $3(13)$ & $3(12.5)$ \\
\hline Hypertension & 0 & 0 & $1(4.3)$ & 0 & 0 & 0 & 0 & 0 & $1(4.3)$ & 0 \\
\hline $\begin{array}{l}\text { Hand-foot } \\
\text { syndrome }\end{array}$ & $1(4.3)$ & 0 & 0 & 0 & 0 & 0 & 0 & 0 & $1(4.3)$ & 0 \\
\hline Fatigue & $3(13)$ & $2(8.3)$ & $2(8.7)$ & $2(8 . .3)$ & 0 & 0 & 0 & 0 & $5(21.7)$ & $4(16.7)$ \\
\hline Proteinuria & $1(4.3)$ & 0 & 0 & 0 & 0 & 0 & 0 & 0 & $1(4.3)$ & 0 \\
\hline Anorexia & $3(13)$ & $2(8.3)$ & $1(4.3)$ & $2(8.3)$ & 0 & 0 & 0 & 0 & $4(17.4)$ & $4(16.7)$ \\
\hline Total & 11(47.8) & $7(29.2)$ & $5(21.7)$ & $5(20.8)$ & 0 & 0 & 0 & 0 & 16(69.6) & $12(50)$ \\
\hline
\end{tabular}

\section{Discussion}

Tumor growth depends on the formation of neovascularization, which has been recognized by cancer biology.Glioma is highly vascular and rich in vascular endothelial growth factor(VEGF) that promotes angiogenesis. The angiogenesis mechanism of glioma may be as follow: tumor growth leads to hypoxia of tumor cells, which causes upregulation of related cytokines, including VEGF, vascular endothelial growth factor receptor (VEGFR), matrix metalloproteinases (MMPs) and hypoxia inducible factor (HIF), etc. These cytokines act on not only vascular endothelial cells but also extracellular matrix to accelerate neovascularization.In consequence, scholars have made a great deal of attempts in the anti-angiogenic treatment of malignant gliomas, and some studies have shown that bevacizumab(BEV) has certain effectiveness in the treatment of malignant gliomas. Therefore, $B E V$, as a humanized monoclonal antibody against VEGF, was approved for the treatment of recurrent GBM by the FDA on May 9, 2009. In 2014, Olivier L. Chinot et alreported a phase III clinical study. The results showed that compared with the radiotherapytemozolomide plus placebo group, the PFS of the radiotherapy-temozolomide plus BEV group was longer (10.6 months vs. 6.2 months; $95 \% \mathrm{Cl} 0.55$ to $0.74 ; \mathrm{P}<0.001)$. There was no significant difference in OS between the two groups $(95 \% \mathrm{Cl} 0.76$ to $1.02 ; \mathrm{P}$ $=0.10)$. However, the occurrence of adverse events of BEV was higher than that of placebo. On the side, the expensive price also limits its clinical use to some extent.

Apatinib is a new type of small molecule anti-angiogenesis inhibitor that can highly selectively compete with the adenosine triphosphate (ATP) binding site of intracellular VEGFR-2 to inhibit the activation of VEGFR-2, thereby blocking downstream signal transduction to control tumor growth. It has been indicated that apatinib can directly promote the apoptosis of tumor cells by inhibiting the PI3K / Akt signaling pathway, up-regulating the expression of Bax and caspase-9 and down-regulating the expression of bcl-2.In addition, apatinib can reverse tumor multidrug resistance by inhibiting the function of various ATP-binding cassette transporter(ABC). This may suggest that the combination of apatinib and traditional chemotherapy drugs can achieve effective results and resist the emergence of multidrug resistance.Some studies have shown that apatinib can not only inhibit the proliferation and colony formation of glioma cells and the invasion of glioma cells by inducing apoptosis, but also promote the TMZ-mediated proliferation inhibition of glioma cells and improve the TMZ-mediated invasion inhibition of glioma cells, thus enhancing the role of $\mathrm{TMZ}$ in glioma cells.

Based on the anti-tumor activity of apatinib and the synergistic anti-tumor effect with TMZ in glioma cells, the following studies were carried out.Wang Yong et alreported a phase II clinical study of apatinib combined with dose-dense TMZ for recurrent glioblastoma.In this clinical study, the objective response rate (ORR) and disease control rate (DCR) were 45\% (9 / 20) and 90\% (18/20), mPFS was 6 months and mOS was 9 months. Another pilot clinical trial study of apatinib combined with irinotecan in the treatment of recurrent high-grade glioma and some other case reports also indicated that apatinib has a certain effect in recurrent 
glioma. It has also been reported that the combination of apatinib and TMZ in the treatment of brainstem glioma has achieved more than 8 months of PFS.

With the concept in mind,we designed such a scheme which combined apatinib with TMZ in concurrent chemoradiotherapy for malignant glioma to carry out exploratory research.

The results showed that compared with the control group, the treatment of malignant glioma in the apatinib group prolonged the PFS by 2.3 months $(p>0.05)$. Although the difference was not statistically significant, the results also showed that the apatinib group significantly improved the cerebral edema of the patients.

Most malignant glioma patients have obvious PTBE, which can cause or aggravate neurological dysfunction and intracranial hypertension,seriously affecting the patients' quality of life and improving the patients' mortality and disability rate.The increase of blood-brain barrier permeability is a key factor in the development of PTBE, and radiotherapy can also damage the vascular endothelium, destroy the blood-brain barrier and aggravate edema.Therefore, for postoperative adjuvant chemoradiotherapy of glioma patients, anti-edema treatment is particularly important.BEV has a certain anti-edema effect, but it also has a large side effect in clinical application. The more common serious side effects are as follows: gastrointestinal perforation; bleeding; arterial thromboembolism, high blood pressure and proteinuria.

The VEGF/VEGFR pathway is closely related to the development of PTBE, among which VEGFR2 mainly plays a role in monitoring the function of vascular endothelium.Compared with other TKI, apatinib shows a strong inhibitory effect on VEGFR2, effectively blocking VEGF / VEGFR2 signal transduction, then reducing vascular permeability and PTBE. Our results are also in accordance with that.

\section{Conclusion}

As an effective and highly selective TKI targeting VEGFR2, apatinib combined with concurrent radiochemotherapy with TMZ for malignant glioma, extended 2.57 months than the median PFS of simple concurrent radiochemotherapy.Although the difference was not statistically significant, the results aiso showed that the combination of apatinib in the treatment of glioblastoma not only significantly improved the patients' cerebral edema, but also had mild adverse reactions. This study has certain clinical significance and can serve as a reference for all physician coping with malignant glioma, which deserves our support for further study in more people.

\section{Declarations}

\section{Authors Contributions Statement}

Xiaoyan Li,Wenmin Ying,Jing Feng and Zhichao Fu conceived and coordinated the study, designed, performed and analyzed the experiments, wrote the paper.Ivjuan Cai,Guo Li,Huachun Luo,Xinpeng Wang and Mengjing Wu carried out the data collection, data analysis, and revised the paper. All authors reviewed the results and approved the final version of the manuscript.

\section{Funding}

This research was no funding.

\section{Disclosure statement}

The author has nothing to disclose.

\section{Ethics and consent to participate declarations}

Patients were not required to given informed consent to the study because the study was a retrospective case-control an analysis that were obtained after each patient agreed to treatment by verbal consent.Individuals can not be identified according to the data presented. 


\section{Conflict-of-interest statement}

The authors report no conflicts of interest.

\section{Data sharing statement}

All data analyzed are included in this study.

\section{References}

1. Ostrom QT,Cioffi G,Gittleman H,Patil N,Waite K,Kruchko C,Barnholtz-Sloan JS.CBTRUS Statistical Report: Primary Brain and Other Central Nervous System Tumors Diagnosed in the United States in 2012-2016.Neuro-oncology.2019;21 Suppl 5:v1v100.

2. Li X, Chen L, et al: CGCG clinical practice guidelines for the management of adult diffuse gliomas. Cancer Lett 375: 263-273, 2016.

3. Stupp R, Hegi ME, Mason WP, van den Bent MJ, Taphoorn MJ, Janzer RC, Ludwin SK, Allgeier A, Fisher B, Belanger K, et al. Effects of radiotherapy with concomitant and adjuvant temozolomide versus radiotherapy alone on survival in glioblastoma in a randomised phase III study: 5-yearanalysis of the EORTC-NCIC trial. Lancet Oncol. 2009; 10:459-466.

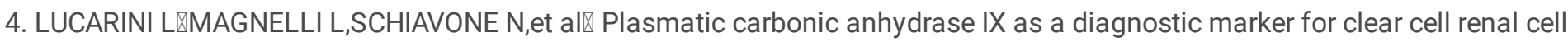

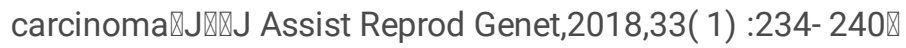

5. SUN S,GONG F,LIN P,et al,Metformin combined with quercetin synergistically repressed prostate cancer cells via inhibition of VEGF/PI3K/Akt signaling pathway \J囚.Gene,2018,664( 8) :50- 57区

6. Ferrara N,Davis-Smyth T. The biology of vascular endothelial growth factor[J]. Endocr Rev,1997,18(1):4 -25.

7. Geng R, Li J. Apatinib for the treatment of gastric cancer. Expert Opin Pharmacother. 2015;16(1):117-122.

8. Folkman J, Bach M, Rowe JW, Davidoff F, Lambert P, Hirsch C, et al. Tumor angiogenesis- therapeuticimplications. New England Journal of Medicine 1971;285(21):1182-6.

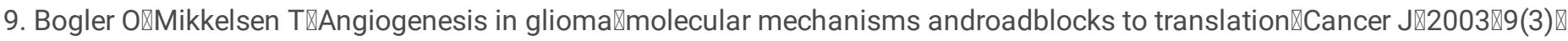
205-1 3区

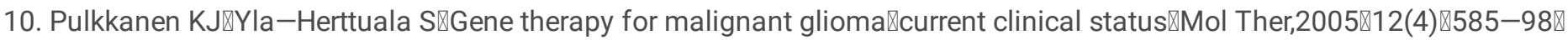

11. Nicholas MK LR囚 Arzbaecher J囚 Paleologos N囚 et al. Bevacizumab in combination with temozolomide in the adjuvant treatment of newly diagnosed glioblastoma multiforme:preliminary results of a phase 2nd study [C]. ASCO Meeting Abstracts $₫ 2009 \otimes 27(15 S): 2016$.

12. Lai $A \rrbracket$ Filka $E \otimes M c G i b b o n ~ B \bigotimes$ et al. Phase 2 nd pilot study of bevacizumab in combination with temozolomide and regional radiation therapy for up-front treatment of patients with newly diagnosed glioblastoma multiforme:interim analysis of safety and tolerability [J]. Int J Radiat Oncol Biol Phys $₫ 2008 \otimes 71(5): 1372-1380$.

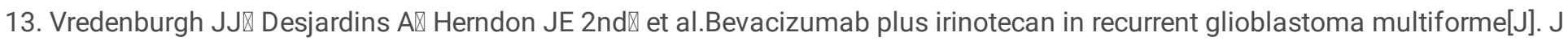
Clin Oncol $82007 \otimes 25(30): 4722-4729$.

14. Cohen MH, Shen YL, Keegan P, Pazdur R. FDA drug approval summary: bevacizumab (Avastin) as treatment of recurrent glioblastoma multiforme. Oncologist. 2009; 14:1131-8.

15. Chinot, O.L.; Wick, W.; Mason, W.; Henriksson, R.; Saran, F.; Nishikawa, R.; Carpentier, A.F.; Hoang-Xuan, K.;Kavan, P.; Cernea, D.; et al. Bevacizumab plus radiotherapy-temozolomide for newly diagnosed glioblastoma.New Engl. J. Med. 2014, 370, 709722.

16. Peng H, Zhang Q, Li J, Zhang N, Hua Y, Xu L, Deng Y, Lai J, Peng Z, Peng B, et al: Apatinib inhibits VEGF signaling and promotes apoptosis in intrahepatic cholangiocarcinoma. Oncotarget 7: 17220-17229, 2016. 13.

17. Kim KL and Suh W: Apatinib, an inhibitor of vascular endothelial growth factor receptor 2, suppresses pathologic ocular neovascularization in mice. Invest Ophthalmol Vis Sci 58: 3592-3599, 2017. 14.

18. Zhang H, Cao Y, Chen Y, Li G, Yu H. Apatinib promotes apoptosis ofthe SMMC-7721 hepatocellular carcinoma cell line via the PI3K/Akt pathway. Oncol Lett. 2018;15(4):5739-5743.

Page $11 / 14$ 
19. Peng $\mathrm{S}$, Zhang $\mathrm{Y}$, Peng $\mathrm{H}$, et al. Intracellular autocrine VEGF signalingpromotes EBDC cell proliferation, which can be inhibited by apatinib. Cancer Lett. 2016;373:193-202.

20. Peng H, Zhang Q, Li J, Zhang N, Hua Y, Xu L. Apatinib inhibits VEGFsignaling and promotes apoptosis in intrahepatic cholangiocarcinomaOncotarget. 2016;7:17220-17229.

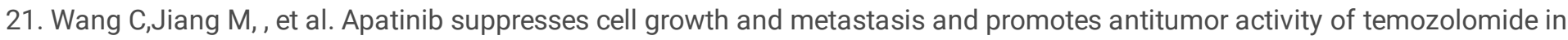
glioma.ONCOLOGY LETTERS 2018;16: 5607-5614.

22. Yong Wang,Xiangji Meng,Shizhen Zhou,Yufang Zhu,Jun Xu,Rongjie Tao.Apatinib Plus Temozolomide for Recurrent Glioblastoma: An Uncontrolled, Open-Label Study.OncoTargets and Therapy.2019;12 10579-10585.

23. Wang L, Liang L, Yang T, et al. A pilot clinical study of apatinib plusirinotecan in patients with recurrent high-grade glioma: clinical trial/experimental study. Medicine (Baltimore). 2017;96:e9053.

24. Zhang H, Chen F, Wang Z, Wu S. Successful treatment with apatinib for refractory recurrent malignant gliomas: a case series. Onco Targets Ther. 2017;10:837-845.

25. Ding X, Sun J, Fan T, Li B. A case report of targeted therapy withapatinib in a patient with recurrent high grade glioma. Medicine (Baltimore). 2018;97:e10859.

26. Yu D, Han G, Liu H, Gao L, Verma V. Treatment of adult brainstem glioma with combined antiangiogenic therapy: a case report and literature review. Onco Targets Ther. 2019;12:1333-1339.

27. Hu WG, Weng YM, Dong Y, Li XP, Song QB. Apatinib in refractory radiationinduced brain edema: A case report.Medicine (Baltimore). 2017 Nov 18;96(46). e7358.

28. Roth P, Regli L, Tonder M, Weller M. Tumor-associated edema in brain cancer patients: pathogenesis and management. Expert RevAnticancer Ther. 2013 Oct 25;13(11):1319-1325.

29. Chinot OL, Wick W, Mason W, Henriksson R, Saran F, Nishikawa R, Carpentier AF, Hoang-Xuan K, Kavan P, Cernea D, et al.Bevacizumab plus radiotherapy-temozolomide for newly diagnosed glioblastoma. N Engl J Med. 2014 Feb 21;370(8):709722.

30. Piazza M, Munasinghe J, Murayi R, Edwards N, Montgomery B,Walbridge S, Merrill M, Chittiboina P. Simulating vasogenic brainedema using chronic VEGF infusionJ Neurosurg.2017 Jan 07;127(4):905-916.

31. Holmes K, Roberts OL, Thomas AM, Cross MJ. Vascular endothelial growth factor receptor-2: structure, function, intracellular signalling and therapeutic inhibition. Cell Signal. 2007 Jul 31;19(10):2003-2012.

32. Yanqiu Song, Bailong Liu, et al. Successful treatment using apatinib in intractable brain edema: A case report and literatures review.CANCER BIOLOGY \& THERAPY.2018; VOL. 19, NO. 12, 1093-1096.

\section{Figures}




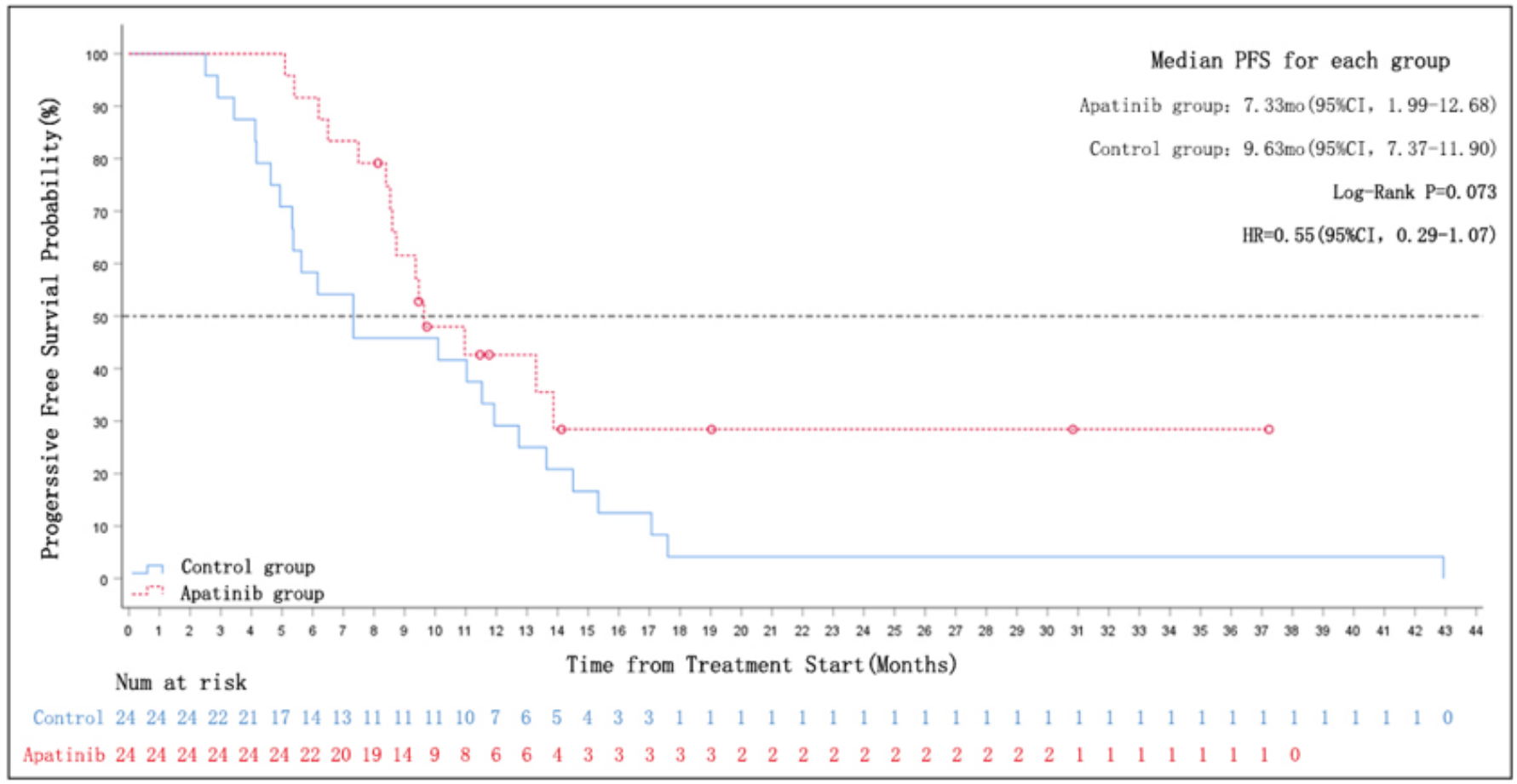

Figure 1

Kaplan-Meier plot of progression-free survival.

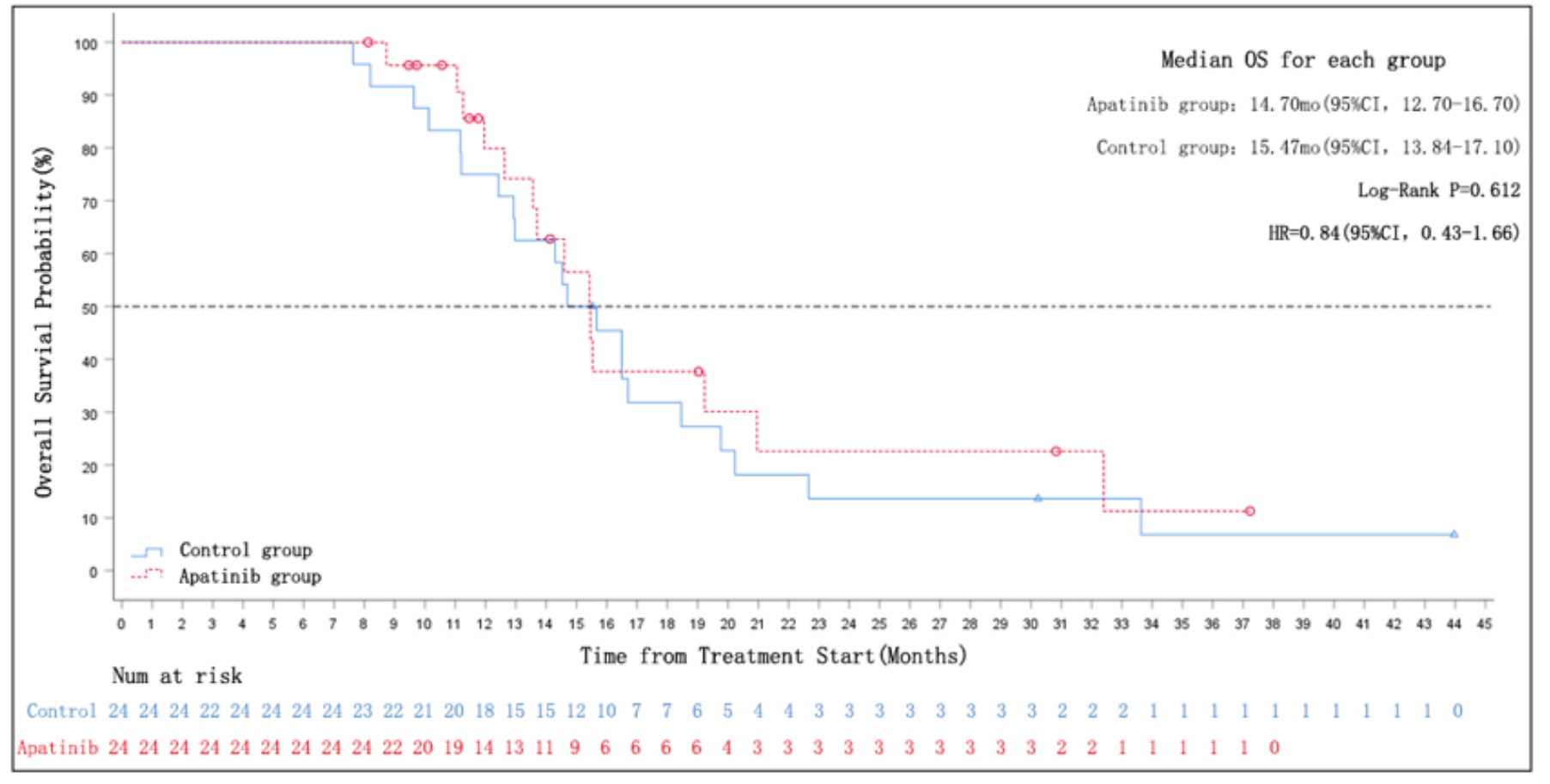

Figure 2

Kaplan-Meier plot of overall survival. 


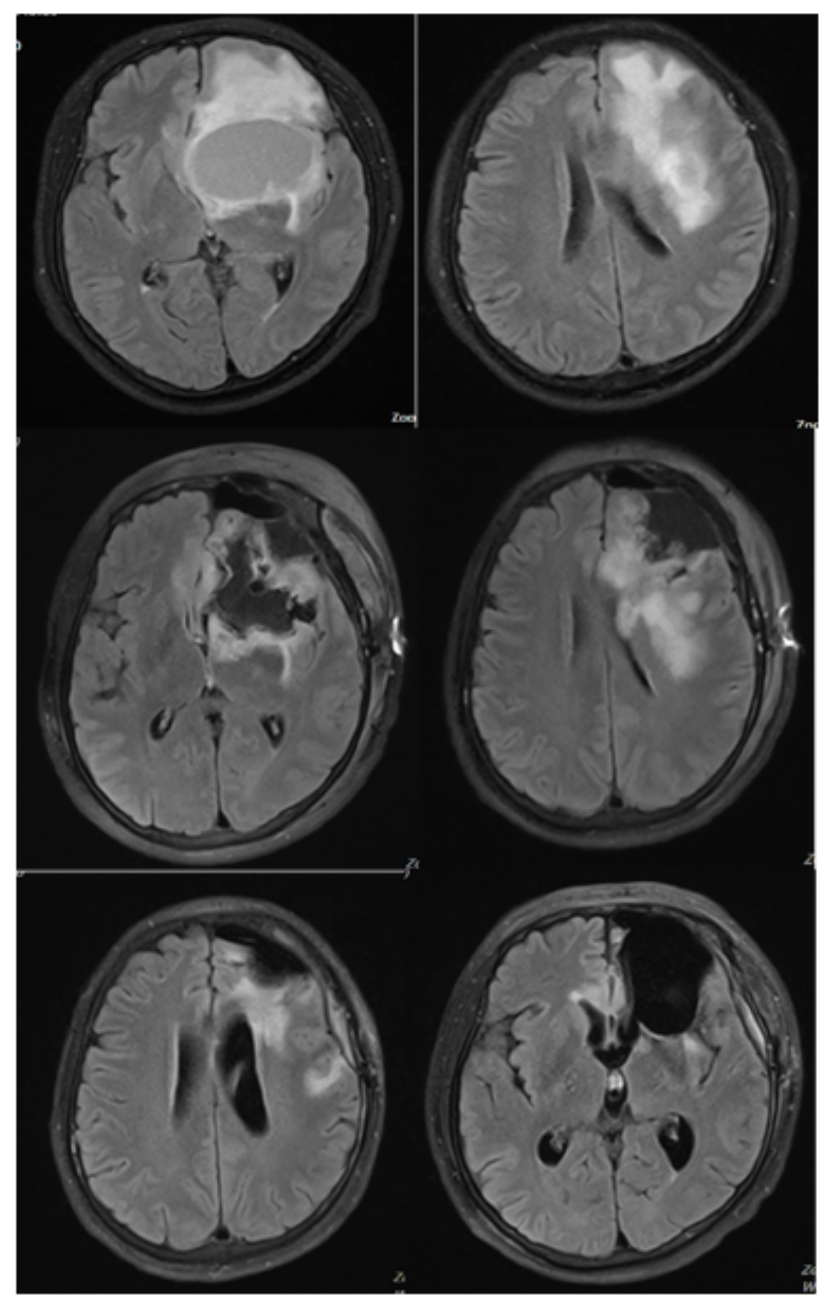

\section{Figure 3}

A typical case of glioblastoma. left: pre-treatment; right: post-treatment MRI examination showed PTBE was significantly smaller after treatment 